\title{
DETERMINAÇÃO CONDUTOMÉTRICA DE CAPTOPRIL EM FORMULAÇÕES FARMACÊUTICAS UTILIZANDO SULFATO DE COBRE(II) COMO TITULANTE
}

Bruna Cláudia Lourenção, Luiz Humberto Marcolino-Junior e Orlando Fatibello-Filho*

Departamento de Química, Universidade Federal de São Carlos, CP 676, 13560-970 São Carlos - SP, Brasil

Recebido em 26/4/07; aceito em 16/7/07; publicado na web em 26/2/08

\begin{abstract}
CONDUCTOMETRIC DETERMINATION OF CAPTOPRIL IN PHARMACEUTICAL FORMULATIONS USING COPPER(II) SULPHATE AS TITRANT. A simple and rapid conductometric method for captopril determination using copper(II) sulphate solution as titrant was developed. The method was based on the chemical reaction between captopril and $\mathrm{Cu}$ (II) ions yielding a precipitate. The conductance of the solution was monitored as a function of the added volume of titrant. The method was applied with success for captopril determination in three pharmaceutical formulations. The relative standard deviation for six successive measurements was smaller than $0.5 \%$. Recovery values from three samples, ranging from 97.7 to $103 \%$, were obtained.
\end{abstract}

Keywords: captopril; conductometric titration; copper (II).

\section{INTRODUÇÃO}

A hipertensão arterial é, atualmente, a doença cardiovascular mais comum, sendo a principal causa de acidente vascular cerebral, podendo conduzir a uma insuficiência cardíaca congestiva, aneurisma dissecante da aorta, infarto do miocárdio e hipertrofia ventricular esquerda ${ }^{1,2}$. Em função de sua alta incidência, tornou-se muito comum na clínica médica a prescrição de um medicamento anti-hipertensivo para uso diário do paciente ${ }^{3}$.

O captopril, 1-[(2S)-3-mercapto-2- metilpropionil]-L-prolina ${ }^{4}$ (Figura 1) é um anti-hipertensivo clássico, cuja ação hipotensora se estabelece basicamente pela inibição da enzima conversora de angiotensina, levando à redução da angiotensina II, que se liga a receptores específicos na membrana celular de vários tecidos, exercendo ação fisiológica de regulação da tensão arterial e sendo um potente vasopressor ${ }^{3}$. Possui um grupo funcional (-SH) que pode agir como um inibidor de radicais livres do organismo com grande eficiência ${ }^{5}$.

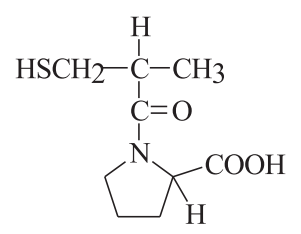

Figura 1. Fórmula estrutural do captopril

Diversos métodos analíticos são descritos na literatura para a determinação quantitativa de captopril em formulações farmacêuticas. Estes procedimentos incluem: espectrofotometria ${ }^{6,7}$, cromatografia líquida de alta eficiência (HPLC) ${ }^{8}$, potenciometria ${ }^{9,10}$, espectrometria de absorção atômica ${ }^{11}$, voltametria ${ }^{4,12-14}$, entre ou$\operatorname{tros}^{15-17}$. Muitos destes métodos não são simples para análises de rotina e requerem instrumentos de alto custo e/ou sofisticados.

O método oficial da Farmacopéia Americana ${ }^{18}$ compreende a titulação iodimétrica de captopril empregando amido como indicador. O método é baseado na mudança de cor da solução, devido à forma- ção do complexo triodeto-amido, o que pode acarretar erros na determinação do ponto de equivalência uma vez que a observação é visual.

Na titulação condutométrica ${ }^{19,20} \mathrm{o}$ aumento ou o decréscimo da condutância são relacionados às variações de concentração das espécies iônicas que participam da reação envolvida. Uma série de medidas da condutância, antes e depois do ponto de equivalência, assinala o ponto final da titulação como uma descontinuidade na variação da condutância.

Há apenas dois métodos condutométricos descritos na literatu$\mathrm{ra}^{21,22}$. Nikolic e Velasevik ${ }^{21}$ determinaram condutometricamente captopril em soluções aquosas. Inicialmente o captopril reage com excesso de solução de nitrato de prata com formação de um sal de prata de baixa solubilidade e ácido nítrico em solução (Equação 1).

$\mathrm{C}_{9} \mathrm{H}_{14} \mathrm{NO}_{3}-\mathrm{SH}+\mathrm{AgNO}_{3} \rightarrow \mathrm{C}_{9} \mathrm{H}_{14} \mathrm{NO}_{3}-\mathrm{SAg}+\mathrm{HNO}_{3}$

Os cátions hidrônios são então determinados por titulação condutométrica, empregando-se solução padronizada de hidróxido de sódio.

Esse procedimento é relativamente moroso e de baixa exatidão, uma vez que na titulação do $\mathrm{H}_{3} \mathrm{O}^{+}$formado (Equação 1) com solução de $\mathrm{NaOH}$ os íons hidrônio dissociados e/ou hidrogênio do grupo carboxílico $\left(\mathrm{pK}_{\mathrm{a} 1}=3,7\right)$ do captopril poderão ser titulados parcialmente.

Outro procedimento empregando titulação condutométrica foi proposto por Mohamed e Aboulenein ${ }^{22}$ para a determinação simultânea de captopril e bendroflumetiazida em preparações farmacêuticas. Nesse procedimento esses fármacos foram solubilizados em dimetilformamida/água 1:1 v/v sendo titulados com solução aquosa de hidróxido de sódio. Apesar destes autores encontrarem recuperações de captopril no intervalo de 98,4 a $101 \%$, a adição do titulante promove a variação da constante dielétrica da mistura de solventes, podendo assim diminuir a exatidão do procedimento.

No presente trabalho descreve-se a titulação condutométrica de captopril com solução padronizada de sulfato de cobre(II) com formação de composto insolúvel deste fármaco com cobre(II), sendo o volume equivalente do titulante (ou teor de captopril na solução do titulado) encontrado no ponto de inflexão da curva condutométrica. 


\section{PARTE EXPERIMENTAL}

\section{Material e equipamentos}

Para as medidas condutométricas utilizou-se uma microbureta automática Methrom/Herisau, modelo E274, um condutivímetro Micronal, modelo B330, célula de vidro com camisa dupla para controle de temperatura e célula condutométrica Digimed DMC-010.

Para o método comparativo utilizou-se um pHmetro Orion, modelo EA 940 com um eletrodo de vidro combinado Analion, modelo $\mathrm{V}$ 620, com um eletrodo de referência de $\mathrm{Ag} / \mathrm{AgCl}$, contendo uma solução de $\mathrm{KCl}$ 3,0 mol L-1.

\section{Soluções e reagentes}

Foram utilizados captopril padrão (Sigma) e $\mathrm{CuSO}_{4} .5 \mathrm{H}_{2} \mathrm{O}$ (Mallinckrodt).

Todos os reagentes empregados foram de grau analítico e todas as soluções foram preparadas com água desionizada obtida de um sistema Milli-Q Millipore (Bedford, MA), modelo UV Plus ultrabaixo teor de substâncias orgânicas dissolvidas.

A solução estoque de $\mathrm{CuSO}_{4} \cdot 5 \mathrm{H}_{2} \mathrm{O} 3,00 \times 10^{-2} \mathrm{~mol} \mathrm{~L}^{-1}$ foi preparada dissolvendo-se uma massa adequada deste sal em água desionizada sendo, então, padronizada por titulação complexométrica com EDTA, de acordo com procedimento proposto na literatura $^{23}$. As soluções de sulfato cúprico em concentrações variando de 5,00 × $10^{-5}$ a $3,00 \times 10^{-2} \mathrm{~mol} \mathrm{~L}^{-1}$ foram obtidas diluindo-se apropriadamente a solução estoque.

As soluções de captopril no intervalo de concentração de 5,00 x $10^{-5}$ a $3,00 \times 10^{-2} \mathrm{~mol} \mathrm{~L}^{-1}$ foram preparadas diluindo-se uma solução estoque do fármaco $1,00 \times 10^{-1} \mathrm{~mol} \mathrm{~L}^{-1}$.

\section{Determinação de captopril em amostras comerciais}

Amostras, na forma de comprimido, contendo captopril foram adquiridas no comércio e analisadas pelo método proposto e os resultados comparados com aqueles obtidos empregando-se um procedimento potenciométrico descrito por Ribeiro e colaboradores 9 .

Dez comprimidos de cada amostra foram triturados e para o preparo da solução foi utilizada uma massa equivalente a um comprimido, que foi dissolvida em $10 \mathrm{~mL}$ de água desionizada e titulada com solução padronizada de $\mathrm{CuSO}_{4} \cdot 5 \mathrm{H}_{2} \mathrm{O}$.

\section{Procedimento comparativo}

Como procedimento comparativo para validar o procedimento proposto empregou-se uma titulação potenciométrica da solução do fármaco com solução de hidróxido de sódio e um eletrodo de vidro combinado como eletrodo indicador ${ }^{9}$.

\section{Titulação condutométrica}

Alíquotas de $10 \mathrm{~mL}$ (soluções de referência ou de amostras) foram colocadas em uma célula de vidro com camisa dupla e tituladas com soluções de mesma concentração de sulfato de cobre, registrando-se a cada adição do titulante, em intervalos de $20 \mathrm{~s}$, o valor de condutância. Esse valor deve ser corrigido em função do volume de titulante adicionado de acordo com a Equação 2, sendo: $\mathrm{L}_{\text {corrig. }}$ a condutância corrigida, $\mathrm{L}_{\text {Exper. }}$ a condutância experimental, $\mathrm{V}_{\mathrm{i}}$ o volume inicial e $\mathrm{V}_{\mathrm{a}}$ o volume adicionado.

$\mathrm{L}_{\text {corrig. }}=\mathrm{L}_{\text {Exp. }} \times\left(\left(\mathrm{V}_{\mathrm{i}}+\mathrm{V}_{\mathrm{a}}\right) / \mathrm{V}_{\mathrm{i}}\right)$
A partir do gráfico de condutância vs. volume de solução de $\mathrm{Cu}^{2+}$, encontrou-se o volume equivalente e, assim, a concentração de captopril nas soluções tituladas.

\section{RESULTADOS E DISCUSSÃO}

\section{Estudos preliminares}

Inicialmente, avaliou-se o intervalo de tempo entre as adições do titulante a fim de se obter maior precisão nos resultados. Os tempos de 15,20 e $30 \mathrm{~s}$ foram estudados utilizando-se concentração de captopril $1,47 \times 10^{-4} \mathrm{~mol} \mathrm{~L}^{-1}$ e de sulfato de cobre $1,16 \times 10^{-4} \mathrm{~mol}$ $\mathrm{L}^{-1}$. O melhor resultado foi obtido com o intervalo de tempo de $20 \mathrm{~s}$ entre as medidas, pois este apresentou um menor desvio padrão relativo e as curvas de titulação, uma melhor resolução.

Com o tempo estabelecido em 20 s, realizaram-se 3 titulações sucessivas de solução padrão de captopril em concentrações variando de 5,00 × $10^{-5}$ a 3,00 × $10^{-2} \mathrm{~mol} \mathrm{~L}^{-1}$ utilizando o titulante sulfato de cobre sempre na mesma concentração do titulado.

A Tabela 1 apresenta os resultados obtidos para cada concentração de solução padrão (de referência) de captopril, podendo ser observada uma boa concordância entre os teores de captopril determinados condutometricamente e as concentrações das soluções de referência.

Tabela 1. Comparação entre os teores conhecidos de captopril na solução de referência e aqueles determinados empregando-se o método condutométrico proposto, a $25^{\circ} \mathrm{C}$

\begin{tabular}{lcc}
\hline $\begin{array}{l}\text { Soluções de referência } \\
\text { de captopril }\left(\mathrm{mol} \mathrm{L}^{-1}\right)\end{array}$ & $\begin{array}{c}\text { Experimental } \\
\left(\mathrm{mol} \mathrm{L}^{-1}\right)\end{array}$ & Erro $(\%)$ \\
\hline $5,10 \times 10^{-5}$ & $(5,06 \pm 0,01) \times 10^{-5}$ & $-0,78$ \\
$1,20 \times 10^{-4}$ & $(1,18 \pm 0,02) \times 10^{-4}$ & $-1,7$ \\
$5,00 \times 10^{-4}$ & $(5,07 \pm 0,02) \times 10^{-4}$ & $+1,4$ \\
$1,20 \times 10^{-3}$ & $(1,22 \pm 0,01) \times 10^{-3}$ & $+1,7$ \\
$3,00 \times 10^{-2}$ & $(2,96 \pm 0,02) \times 10^{-2}$ & $-1,4$ \\
\hline
\end{tabular}

* $\mathrm{n}=3$; média \pm desvio padrão

Para concentrações de captopril menores de 5,00 x $10^{-5} \mathrm{~mol} \mathrm{~L}^{-1}$, a adição do titulante ocasionou pequenas variações de condutância. Como consequiência, a determinação do ponto de equivalência pode ser determinada até um teor mínimo de $3,00 \times 10^{-5} \mathrm{~mol} \mathrm{~L}^{-1}$ mas com uma exatidão menor, da ordem de $8-9 \%$.

A Figura 2 apresenta uma curva condutométrica característica obtida para a determinação de $5 \mathrm{~mL}$ de captopril $1,20 \times 10^{-3} \mathrm{~mol} \mathrm{~L}^{-1}$ utilizando solução de $\mathrm{Cu}^{2+} 1,16 \times 10^{-3} \mathrm{~mol} \mathrm{~L}^{-1}$ como titulante.

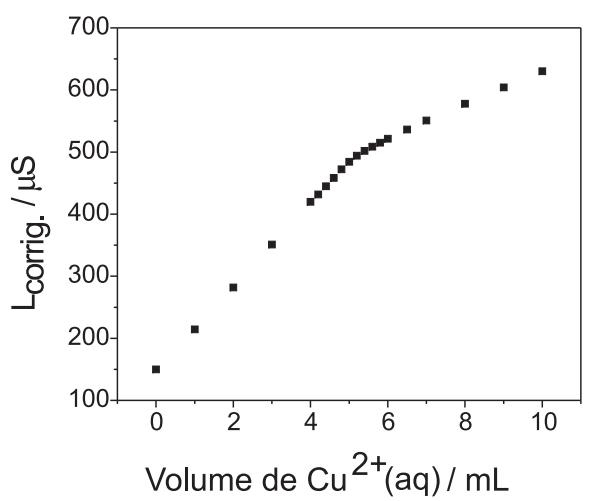

Figura 2. Curva característica da titulação condutométrica de captopril 1,20 $\times 10^{-3} \mathrm{~mol} \mathrm{~L}^{-1} \mathrm{com} \mathrm{CuSO} \cdot 1,16 \times 10^{-3} \mathrm{~mol} \mathrm{~L}^{-1}$ 
A condutância medida antes da adição do titulante (volume de $\mathrm{Cu}^{2+}$ igual a zero) deve-se à dissociação do próton do grupo carboxílico e, em menor intensidade, do próton do grupo sulfidrila presente na molécula de captopril (Figura 1).

Até o ponto de equivalência, a titulação envolve a gradual substituição de dois prótons da molécula de captopril por um átomo de $\mathrm{Cu}^{2+}$ (Figura 3). Este aumento da condutância é devido à maior mobilidade do íon $\mathrm{H}_{3} \mathrm{O}^{+}$em relação àquela do $\mathrm{Cu}^{2+}$, ocasionando assim uma forte inclinação da curva condutométrica (primeiro ramo da curva).

$$
\text { (aq) }
$$

Figura 3. Reação envolvida na titulação condutométrica do captopril com sufalto de cobre(II)

Após o ponto de equivalência, à medida que são adicionados volumes crescentes da solução do titulante, há um aumento linear da condutância em função do volume da solução de $\mathrm{Cu}^{2+}$ adicionada, devido ao aumento das concentrações desse cátion metálico e dos ânions $\mathrm{SO}_{4}^{2-}$ na solução resultante. Como a soma das mobilidades desses íons é menor que aquela dos cátions $\mathrm{H}_{3} \mathrm{O}^{+}$há uma diminuição da inclinação do segundo ramo da curva de titulação, sendo o ponto de equivalência determinado pelo ponto de intersecção dos dois segmentos de reta.

No estudo da repetibilidade do procedimento condutométrico, uma solução de captopril $1,20 \times 10^{-3} \mathrm{~mol} \mathrm{~L}^{-1}$ foi titulada condutometricamente por seis vezes $(\mathrm{n}=6)$ com solução de $\mathrm{Cu}^{2+} 1,20 \times 10^{-3} \mathrm{~mol} \mathrm{~L}^{-1} \mathrm{e}$ o desvio padrão relativo (RSD) obtido foi menor que $0,5 \%$.

\section{Estudo de interferentes em potencial e teste de recuperação}

O efeito de interferentes em potencial na determinação de captopril em formulações farmacêuticas foi avaliado para excipientes normalmente presentes em amostras comerciais (amido, lactose, celulose, dióxido de silício e estearato de sódio).

Nesses experimentos foram feitas comparações do volume do ponto de equivalência obtido empregando-se uma solução de referência de captopril $1,0 \times 10^{-3} \mathrm{~mol} \mathrm{~L}^{-1} \mathrm{com}$ o volume do ponto de equivalência obtido empregando-se a mesma solução de referência de captopril juntamente com cada um dos interferentes em potencial nas concentrações esperadas nos produtos comerciais. Não houve interferência significativa nos valores de volume de equivalência en- contrados para essas substâncias estudadas. Cabe salientar que a maioria destes excipientes é insolúvel e/ou possui baixa solubilidade.

A Tabela 2 apresenta os resultados obtidos no estudo de adição e recuperação do analito em três diferentes concentrações de captopril, adicionadas a três diferentes amostras de formulações farmacêuticas. Os resultados são comparados com aqueles obtidos com as amostras não adicionadas de padrão. As recuperações de

\begin{tabular}{|c|c|c|c|}
\hline \multirow[t]{2}{*}{ Amostra } & \multicolumn{2}{|c|}{ Teor de Captopril (mg mL $\left.\mathrm{m}^{-1}\right)$} & \multirow{2}{*}{$\begin{array}{c}\text { Recuperação } \\
\text { E } \%\end{array}$} \\
\hline & adicionado & determinado & \\
\hline \multirow[t]{3}{*}{ Genérico } & 1,09 & $1,12 \pm 0,01$ & 103 \\
\hline & 1,64 & $1,61 \pm 0,01$ & 98,2 \\
\hline & 2,18 & $2,14 \pm 0,02$ & 98,2 \\
\hline \multirow[t]{3}{*}{ Captotec } & 1,09 & $1,08 \pm 0,03$ & 99,1 \\
\hline & 1,64 & $1,63 \pm 0,04$ & 99,4 \\
\hline & 2,18 & $2,19 \pm 0,03$ & 100 \\
\hline \multirow[t]{3}{*}{ Capoten } & 1,09 & $1,08 \pm 0,02$ & 99,1 \\
\hline & 1,64 & $1,61 \pm 0,01$ & 98,2 \\
\hline & 2,18 & $2,13 \pm 0,01$ & 97,7 \\
\hline
\end{tabular}
captopril variaram de 97,7 a 103\%, evidenciando assim que não há interferência significativa da matriz da amostra no procedimento condutométrico proposto.

Tabela 2. Estudo de adição e recuperação de captopril*

$\mathrm{n}=3$

\section{Aplicações}

O método proposto foi aplicado na determinação de captopril em três diferentes formulações farmacêuticas e os resultados comparados com aqueles obtidos empregando-se um método comparativo9. A Tabela 3 apresenta os resultados obtidos nestas determinações. Como pode ser observado, o procedimento proposto possui boa precisão sendo que os valores encontrados empregando-se ambos os métodos estão em boa concordância a um nível de confiança de $95 \%\left(\mathrm{t}_{\text {calc }}=2,31\right.$ e $\left.\mathrm{t}_{\text {crítico }}=4,30\right)$.

\section{CONCLUSÕES}

O método condutométrico proposto para a determinação de captopril em produtos farmacêuticos é simples, preciso, exato, de baixo custo e não necessita de nenhum tipo de tratamento da amostra, podendo ser aplicado em laboratórios de análise de rotina.

\section{AGRADECIMENTOS}

A o apoio financeiro do CNPq, da FAPESP e CAPES, bem como à FAPESP pela bolsa de iniciação científica de B. C. Lourenção (Proc. no. 06/05693-0) e à CAPES pela bolsa de doutorado de L. H. Marcolino Jr.

Tabela 3. Teores de captopril obtidos empregando-se titulação potenciométrica ${ }^{9}$ e titulação condutométrica

\begin{tabular}{lcccc}
\hline Amostra & Rotulado & $\begin{array}{c}\text { Teor }(\mathrm{mg} / \text { comprimido }) \\
\text { Titulação } \\
\text { Potenciométrica }\end{array}$ & $\begin{array}{c}\text { Titulação } \\
\text { Condutométrica }\end{array}$ & Erro $_{1}(\%)$ \\
\hline Genérico $^{\circledR}$ & 25,0 & $24,8 \pm 0,1$ & $25,1 \pm 0,3$ & Erro $_{2}(\%)$ \\
Captotec $^{\circledR}$ & 12,5 & $12,1 \pm 0,1$ & $12,5 \pm 0,1$ & $+1,2$ \\
Capoten $^{\circledR}$ & 12,5 & $12,7 \pm 0,3$ & $12,6 \pm 0,3$ & 0 \\
\hline
\end{tabular}

Erro $_{1}$ : proposto vs. rotulado. Erro ${ }_{2}$ : proposto vs. comparativo 


\section{REFERÊNCIAS}

1. Oates A. J. Em Fármacos anti-hipertensivos e a terapia farmacológica da hipertensão: as bases farmacológicas da terapêutica; Hardman, J. G.; Limbird, L. E., eds.; $9^{a}$ ed., McGraw-Hill: México-DF, 1996.

2. Jackson, E. K.; Garrison, J. C. Em Renina angiotensina: as bases farmacológicas da terapêutica; Hardman, J. G.; Limbird, L. E., eds.; $9^{a}$ ed., McGraw-Hill: México-DF 1996.

3. Sucar, D. D.; Rev. Bras. Psiquiatr. 2000, 22, 134

4. Parham, H.; Zargar, B.; Talanta 2005, 65, 776.

5. Shahrokhian, S.; Karimi, M.; Khajehsharifi, H.; Sens. Actuators, B 2005 , 109, 278.

6. Tzanavaras, P. D.; Themelis, D. G.; Economou, A.; Theodoridis, G.; Microchim. Acta 2003, 142, 55.

7. Shama, S. A.; Amin, A. E.; Omara, H.; J. Quant. Spectrosc. Radiat. Transfer 2006, 102, 261.

8. Mirza, T.; Tan, H. S. I.; J. Pharm. Biomed. Anal. 2001, 25, 39.

9. Ribeiro, P. R. da S.; Santini, A. O.; Pezza, H. R.; Pezza, L.; Eclética Química 2003, 28, 39.

10. Pimenta, A. M.; Araújo, A. N.; Montenegro, M. C. B. S. M.; Anal. Chim. Acta 2001, 438, 31.
11. El- Reis, M. A.; Abou-Attia, F. M.; Kenawy, I. M. M.; J. Pharm. Biomed. Anal. 2000, 23, 249

12. Siangproh, W.; Ngamukot, P.; Chailapakul, O.; Sens. Actuators, B 2003, 91, 60.

13. Ioannides, X.; Economou, A.; Voulgaropoulos, A.; J. Pharm. Biomed. Anal. 2003, 33, 309

14. Shahrokhian, S.; Karimi, M.; Khajehsharifi, H.; Anal. Chim. Acta 2005, 546, 60.

15. Palomeque, M. E.; Fernández-Band, B. S.; J. Pharm. Biomed. Anal. 2002, $30,547$.

16. Du, J.; Li, Y.; Lu, J.; Luminescence 2002, 17, 165.

17. Ma, W. X.; Spectroscopy and Spectral Analysis 1999, 19, 118.

18. The United States pharmacopeia; The National Formulary, US Pharmacopeial Convention: captopril, Rockville MD, 24 ${ }^{\text {th }}$ ed., 2000, p. 296.

19. Ohlweiler, O. A.; Fundamentos de análise instrumental, LTC: Rio de Janeiro, 1981

20. Ohlweiler, O. A.; Química analítica quantitativa, $2^{\mathrm{a}}$ ed., LTC: Rio de Janeiro, 1980

21. Nikolic, K.; Velasevic, K.; Pharmazie 1989, 44, 155.

22. Mohamed, M. E.; Aboulenein, H. Y.; Anal. Lett. 1985, 18, 2591.

23. MERCK. Métodos complexométricos de valoración con Titriplex, $3^{\mathrm{a}}$ ed., Darmstadt, E. Merck, 1972, p 33-34. 